\title{
Association of neuropsychiatric syndromes with global clinical deterioration in Alzheimer's disease patients
}

\author{
Florindo Stella, ${ }^{1,2}$ Jerson Laks, ${ }^{3}$ José Sílvio Govone, ${ }^{4}$ Kate de Medeiros ${ }^{5}$ \\ and Orestes Vicente Forlenza ${ }^{2}$ \\ ${ }^{1}$ Biosciences Institute, Campus of Rio Claro-SP, UNESP - Universidade Estadual Paulista, Rio Claro, SP, Brazil \\ ${ }^{2}$ Laboratory of Neurosciences (LIM-27), Department and Institute of Psychiatry, Faculty of Medicine, University of Sao Paulo, Sao Paulo, SP 05403-010, \\ Brazil \\ ${ }^{3}$ Center for Alzheimer's Disease and Related Disorders, Institute of Psychiatry, Federal University of Rio de Faneiro-RF, Brazil; and Center for Studies and \\ Research on Aging, Institute Vital Brazil, Rio de Faneiro-RF, Brazil \\ ${ }^{4}$ Department of Statistics, Applied Mathematics, and Computer Sciences, UNESP - Universidade Estadual Paulista; and Center of Environmental Studies \\ (CEA), Institute of Geosciences and Exact Sciences, Rio Claro, SP, Brazil \\ 5 Department of Sociology and Gerontology, Miami University, Oxford, Ohio, USA
}

\section{ABSTRACT}

Background: Data on the relationship between behavioral disturbances in Alzheimer's disease (AD) and global clinical deterioration is still controversial. The purpose of this study was to explore potential correlations of neuropsychiatric syndromes with global clinical deterioration in patients with AD, with particular consideration on severity levels of dementia.

Methods: AD patients $(n=156)$ aged 76.7 years from Brazilian clinical centers were assessed to diagnose the five neuropsychiatric syndromes measured by the Neuropsychiatric Inventory-Clinician rating scale (NPI-C): psychosis, agitation, affective, apathy, and sleep. These syndromes were then analyzed for their correlation with the Global Deterioration Scale (GDS). To analyze the association of neuropsychiatric syndromes with the GDS, considering the total sample and patients grouped by dementia severity levels, we applied the coefficient of multiple correlation (Ryy), adjusted multiple linear regression, and the coefficient of determination $\left(R^{2} y x\right)$. We tested the significance of correlation coefficients using the Student t-test for simple correlations (a single independent variable) and analysis of variance (ANOVA) for multiple correlations. ANOVA was also used to compare means of demographic and some clinical variables at different levels of dementia.

Results: For the total sample, apathy and agitation syndromes were most strongly correlated $(0.74 ; 0.72$, respectively) with clinical deterioration according to the GDS, followed by psychosis (0.59), affective (0.45), and sleep syndromes (0.34). Agitation significantly correlated with mild and moderate dementia (CDR 1: 0.45; and CDR 2: 0.69, respectively). At CDR 2, agitation and affective syndromes were most strongly correlated $(0.69 ; 0.59$, respectively) with clinical deterioration while at $\operatorname{CDR} 3$, the apathy syndrome was most strongly correlated with clinical deterioration (0.52).

Conclusions: Agitation, apathy, and affective disorders were the syndromes most strongly correlated with global deterioration in $\mathrm{AD}$ patients, becoming more evident at severe stages of dementia.

Key words: neuropsychiatric syndromes, global deterioration, Alzheimer's disease, dementia

\section{Introduction}

With the aim of appropriately recognizing and treating behavioral disturbances in $\mathrm{AD}$ patients, several researchers have grouped these

Correspondence should be addressed to: Florindo Stella, UNESP - Universidade Estadual Paulista, Biosciences Institute, Campus of Rio Claro-SP; and Laboratory of Neurosciences, Institute and Department of Psychiatry, Faculty of Medicine, University of São Paulo-SP, Brazil. Phone: 551935264245; Fax: 551935264246. Email: fstella@rc.unesp.br. Received 9 Aug 2015; revision requested 8 Sep 2015; revised version received 1 Nov 2015; accepted 6 Nov 2015. First published online 17 December 2015. symptoms into distinct syndromes using different methodological designs and based on various theoretical issues (Lyketsos et al., 2002; Aalten et al., 2005; Dechamps et al., 2008; Spalletta et al., 2010; Lyketsos and Miller, 2012). For instance, the occurrence of overlapping symptoms among several syndromes is not uncommon. Although agitated behavior has been included in the "agitation syndrome," this behavior may also be present also in "sleep disorders syndrome" or in "psychotic syndrome" (Aalten et al., 2008; Cummings et al., 2015). Apathetic symptoms have been classified 
as an "apathy syndrome," characterized by loss of motivation related to reduced cognitive interest, diminished initiative, and emotional blunting; however, depending on particular features from the clinical profile or emotional suffering, these symptoms might be part of depression (Holthoff et al., 2005; Robert et al., 2009; Lyketsos et al., 2011; Chi et al., 2014; Cummings et al., 2015).

Authors have proposed different ways to classify neuropsychiatric symptoms of $\mathrm{AD}$ into selected syndromes such as mood, psychotic, and frontal manifestations (Frisoni et al., 1999); hyperactivity, psychosis, anxiety, mood-excitement, and mooddepression-apathy (Spalletta et al., 2004); affect, physical behavior, psychosis, and hypomania (Mirakhur et al., 2004); as well as psychotic, affective, and behavior syndromes (Garre-Olmo et al., 2010), among others. However, a certain degree of concordance of correlated symptoms is required for feasible clustering neuropsychiatric symptoms into single syndromes. An investigation involving data from several studies used factor analysis to identify and group related neuropsychiatric symptoms into distinct syndromes, such as hyperactivity/agitation behaviors, psychosis, affective manifestations, and apathy. However, behaviors such as aberrant motor behavior and night-time disturbances have no sufficient methodological consistency to be categorized as a single syndrome (Aalten et al., 2005).

Other examples of grouping include delusions and hallucinations which have been regularly grouped under the label of "psychotic syndrome"; depression and anxiety in general have been grouped into "affective syndrome"; agitated behavior, aberrant motor behavior, and aggression have been reported as "hyperactivity" or "agitation syndrome"; sleep disorders and nighttime behaviors have been considered as "sleep disorders syndrome" (Aalten et al., 2008; GarreOlmo et al., 2010; Lyketsos and Miller, 2012).With the goal of better understanding, treating, or eventually preventing behavioral disturbances in dementia, an international consensus was led by C.G. Lyketsos and D.S. Miller for the Neuropsychiatric Syndromes Professional Interest Area of the International Society to Advance Alzheimer's Research and Treatment (Lyketsos and Miller, 2012). This consensus proposed specific recommendations for advances in research and improvement of neuropsychiatric symptoms detection, as well as dissemination of effective care for patients. This consensus also recommended grouping consistently correlated symptoms into distinct syndromes and that such grouping should be considered in research on and interventions for $\mathrm{AD}$ and other dementias.
Despite these efforts, associations between neuropsychiatric syndromes and global clinical deterioration according to severity levels of dementia represent an issue to be examined in $\mathrm{AD}$ patients. To address the methodological weaknesses in other studies which have attempted to group neuropsychiatric behavioral patterns of $\mathrm{AD}$, our aim was to explore potential correlations of neuropsychiatric syndromes with global clinical deterioration, with particular concern regarding severity levels of dementia.

\section{Methods}

\section{Patients}

The study comprised 156 eligible $\mathrm{AD}$ patients aged $76.7( \pm 7.2)$ years, with $5.5( \pm 4.3)$ years of education on average. All patients were recruited from five Brazilian centers which regularly diagnose and treat people with dementia.

Probable AD was diagnosed according to the criteria established by the National Institute of Neurological and Communicative Disorders and Stroke-Alzheimer's Disease and Related Disorders Association (NINCDS-ADRDA) (McKhann et al., 1984). Clinical diagnosis of dementia included the criteria fulfillment recommended by the Diagnostic and Statistical Manual of Mental Disorders, 4th edition-revised (American Psychiatric Association, 2000). The raters determined the dementia severity using the Mini-Mental State Examination (MMSE; Folstein et al., 1975), the Clinical Dementia Rating (CDR; Hughes et al., 1982), the Pfeffer Functional Activities Questionnaire (Pfeffer et al., 1982), and the GDS (Reisberg et al., 1982). The GDS comprises behavioral characteristics manifested by patients at distinct levels of dementia severity (Reisberg et al., 1982). The scales applied in the present study were already validated for the Brazilian community or have been extensively used in patients with dementia in our country. Table 1 summarizes demographic and selected clinical features.

The inclusion criteria were as follows: the presence of a knowledgeable informant (caregiver or family member) able to identify and describe neuropsychiatric symptoms in the patient occurring during the past month, as well as maintaining regular contact with the patient at least three times per week over the past three months.

Respective ethics review boards from each research center approved the study, and family members or legal representatives of patients signed written informed consent. The research was conducted according to the principals of the Helsinki Declaration. 
Table 1. Selected demographic and clinical variables that were compared with dementia severity levels using one-way ANOVA

\begin{tabular}{|c|c|c|c|c|c|c|}
\hline & DEMOGRAF & IC AND SE & CTED CLINIC & FEATURES & & \\
\hline $\begin{array}{l}\text { DEMENTIA } \\
\text { SEVERITY }\end{array}$ & PATIENTS & $\begin{array}{l}\text { AGE } \\
\text { (YEARS) }\end{array}$ & $\begin{array}{l}\text { EDUCATION } \\
\text { (YEARS) }\end{array}$ & MMSE & PFEFFER & GDS \\
\hline CDR 1 (mild) & $60(\mathrm{~F}: 42 / \mathrm{M}: 18)$ & $77.4 \pm 6.1$ & $5.9 \pm 3.9$ & $22.8 \pm 3.5$ & $8.3 \pm 6.3$ & $2.7 \pm 0.5$ \\
\hline CDR 2 (moderate) & $53(\mathrm{~F}: 44 / \mathrm{M}: 9$ & $76.8 \pm 7.1$ & $5.8 \pm 4.9$ & $17.4 \pm 3.3$ & $18.3 \pm 6.1$ & $4.3 \pm 0.7$ \\
\hline CDR 3 (severe) & $43(\mathrm{~F}: 29 / \mathrm{M}: 14)$ & $75.6 \pm 8.6$ & $4.4 \pm 3.8$ & $9.2 \pm 5.3$ & $26.2 \pm 4.7$ & $6 \pm 0.7$ \\
\hline Total & $156(\mathrm{~F}: 115 / \mathrm{M}: 41)$ & $76.7 \pm 7.2$ & $5.5 \pm 4.3$ & $17.2 \pm 6$ & $16.6 \pm 9.3$ & $4.2 \pm 1.4$ \\
\hline$p$-value & - & 0.45 & 0.15 & $<0.0001^{*}$ & $<0.0001^{*}$ & $<0.0001^{*}$ \\
\hline
\end{tabular}

CDR: Clinical dementia rating. ${ }^{*}$ Significant differences at $1 \%$ of probability.

MMSE: Mini-Mental State Examination; Pfeffer: Pfeffer Functional Activities Questionnaire; GDS: Global Deterioration Scale; CDR:

Clinical Dementia Rating; F: female; M: male.

\section{Procedures}

The patients were classified into mild (CDR $1, n=$ 60 ), moderate (CDR 2, $n=53$ ), and severe (CDR $3, n=43$ ) dementia. To estimate neuropsychiatric syndromes, eligible clinician raters specifically trained to use the NPI-C (de Medeiros et al., 2010) interviewed each informant/patient dyad. The NPI-C was already validated for the Brazilian community. Methodological procedures comprised convergent validity to estimate correlations between NPI-C domains and selected scales that measure the same symptoms; these procedures also included inter-rater reliability since independent raters administered the scale (Stella et al., 2013). To complete the NPI-C, the rater first asked caregivers or family members about the frequency and severity of each item in each domain and their distress level due to the patient's behavioral disturbances. When possible, the rater interacted with the patient to help the rater gain first-hand knowledge of the patient and his or her neuropsychiatric symptoms. Based on caregiver reports, patient interview or observation, and any additional information on clinically relevant data (for instance, a particular description from the patient record), the rater appropriately determined the final score for each symptom in each domain of the NPI-C. Afterwards, considering all available relevant information and according to his or her clinical judgment, the rater estimated the severity of each neuropsychiatric domain.

\section{Classification of neuropsychiatric symptoms into distinct syndromes}

In the present study, we used the classification of neuropsychiatric syndromes recommended by the Neuropsychiatric Syndromes Professional Interest Area of the Alzheimer's Association International Society to Advance Alzheimer's Research and Treatment (Lyketsos and Miller, 2012). This group proposed grouping neuropsychiatric symptoms observed in patients with $\mathrm{AD}$ or other dementias into five syndromes using NPI-C domains (listed within the parenthesis): (1) psychosis (delusions, hallucinations); (2) agitation (agitated behavior, aggression, disinhibition, irritability, euphoria, aberrant motor behavior, aberrant vocalizations); (3) affective disorder (depression, anxiety); (4) apathy (indifference, apathetic symptoms, appetite eating disorders); and (5) sleep disorders (night-time behavior disturbances). Overall, this approach provides a strong theoretical basis and methodological approach to better understanding neuropsychiatric symptoms.

\section{Statistical analyses}

Descriptive analyses were performed to determine the demographic and clinical features of patients. The conditions for the use of statistical analyses (normality, homoscedasticity, and independence) were preliminarily verified. Correlations were calculated (Pearson's correlation coefficient) between neuropsychiatric syndromes and GDS according to dementia severity levels (CDR 1, 2, and 3) and the significance of these correlations was verified (Student's t test). To analyze the association between neuropsychiatric syndromes and GDS scores in all of the patients together $(n=156)$, we applied the coefficient of multiple correlation (Ryy). To estimate if neuropsychiatric syndromes could interfere with GDS scores we used adjusted multiple linear regression, as well as the coefficient of determination $\left(\mathrm{R}^{2} \mathrm{yx}\right)$. The same procedure was applied to patients grouped by dementia severity levels (CDR 1, 2, and 3). To estimate if the variability of neuropsychiatric syndromes could significantly interfere with GDS scores, we used the Student's t test. We inserted additional analyses involving correlations of GDS scores with the Pfeffer Functional Activities Questionnaire and 


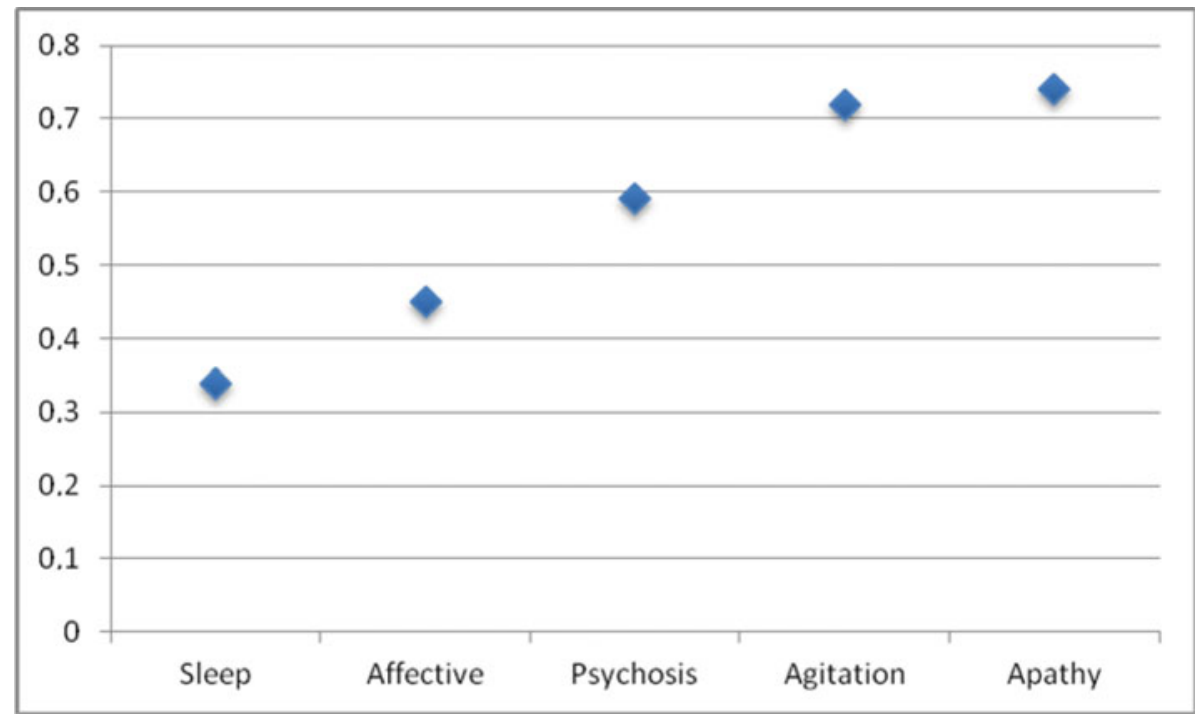

Figure 1. (Colour online) Correlation of neuropsychiatric syndromes with GDS (total sample; $n=156)$. GDS: Global Deterioration Scale.

with demographic data. To compare the continuous variables of mean age, years of education, and clinical variables such as dementia severity levels according to the CDR, we applied one-way ANOVA. For variables with significant differences when compared by CDR levels, the Tukey's test was applied to calculate the degree of averages from each other. To complete the analyses of clinical variables, we compared the means of the MMSE to severity levels of dementia, and as expected, with significant differences $(p<0.0001)$ among CDR 1 versus CDR 2; CDR 1 versus CDR 3; and CDR 2 versus $C D R$ 3. Likewise, we compared the means from the Pfeffer Functional Activities Questionnaire and GDS scores at distinct levels of the CDR, and we detected significant differences from each other $(p<0.0001)$. All statistical analyses were performed with significance level of $p<0.05$.

\section{Results}

Figure 1 depicts the main correlations of neuropsychiatric syndromes with global deterioration according to the GDS scores for all patients. For the total sample, apathy and agitation syndromes were significantly correlated (respectively $0.74 ; 0.72 ; p$ $<0.0001$ ) with clinical deterioration according to the GDS, followed by psychosis (0.59), affective syndromes (0.45), and subsequently by sleep disorders syndrome (0.34). (See Table 2).

Summarizing the results, the agitation syndrome correlated with the GDS at CDR 1 (0.45), at CDR 2 (0.69), and at CDR 3 (0.43); the affective syndrome correlated with the GDS at CDR 2 (0.59); and the apathy syndrome correlated with the GDS at CDR 2 (0.52). (See Tables 2 and 3).
At moderate severity (CDR 2), the agitation syndrome (0.69) and affective syndrome (0.59) significantly interfered with GDS scores. Likely, the apathy syndrome contributed to high GDS scores from patients at severe dementia (CDR 3; 0.52 ). Table 3 displays these data. Analyses of other variables from all patients together showed high correlation of functional activities, measured by the Pfeffer questionnaire, with GDS scores (0.74; Ryy).

\section{Discussion}

It is generally accepted that individual symptoms tend to have intermittent course throughout the time and disease aggravation (Cerejeira et al., 2012). Likewise, neuropsychiatric syndromes in $\mathrm{AD}$ follow a course of changing prevalence through the disease progression, particular psychosocial determinants, and specific neurobiological correlates (Robert et al., 2005; Lyketsos et al., 2011). Furthermore, these syndromes in $\mathrm{AD}$ have received notable exploration by recent investigations (Gonfrier et al., 2012; Brodaty et al., 2015; Cummings et al., 2015), even in early stages of the disease (Van der Mussele et al., 2015).

We examined whether five neuropsychiatric syndromes of $\mathrm{AD}$ patients were correlated with global clinical deterioration according to severe levels of dementia. Overall, our results show that the neuropsychiatric syndromes were closely related to global deterioration measurements. In fact, the agitation and apathy syndromes exhibited strong association with GDS scores. According to the dementia severity, the mild stage (CDR 1) moderately correlated with the agitation syndrome; at CDR 2, the agitation syndrome and the affective syndrome 
Table 2. Significant correlations of selected neuropsychiatric syndromes with the global deterioration scale (GDS) from all patients $(n=156)$ (Student's t-test)

\begin{tabular}{|c|c|c|c|}
\hline CHARACTERISTICS & $\begin{array}{l}\text { SYNDROME } \\
\text { VERSUS GDS }\end{array}$ & $\begin{array}{l}\text { CORRELATIONS } \\
\text { (RYY) ( } p \text { VALUES) }\end{array}$ & $\begin{array}{l}\text { PERCENTAGE OF } \\
\text { COEFFICIENT OF } \\
\text { DETERMINATION }\left(R^{2} \%\right)\end{array}$ \\
\hline \multirow{5}{*}{$\begin{array}{l}\text { All patients }(n=156) \text { : } \\
\quad \text { (CDR 1, mild, } n=60 ; \\
\text { CDR 2, moderate } n=53 \text {; } \\
\text { CDR 3, severe, } n=43 \text { ) }\end{array}$} & Apathy & $0.74(<0.0001)$ & 54.9 \\
\hline & Agitation & $0.72(<0.0001)$ & 51.3 \\
\hline & Psychosis & $0.59(<0.0001)$ & 34.8 \\
\hline & Affective & $0.45(<0.0001)$ & 20.2 \\
\hline & Sleep disorders & $0.34(<0.0001)$ & 11.5 \\
\hline
\end{tabular}

CDR: Clinical dementia rating; GDS: Global Deterioration Scale.

Table 3. Significant correlations of selected neuropsychiatric syndromes with the global deterioration scale according to dementia severity, as well as other data

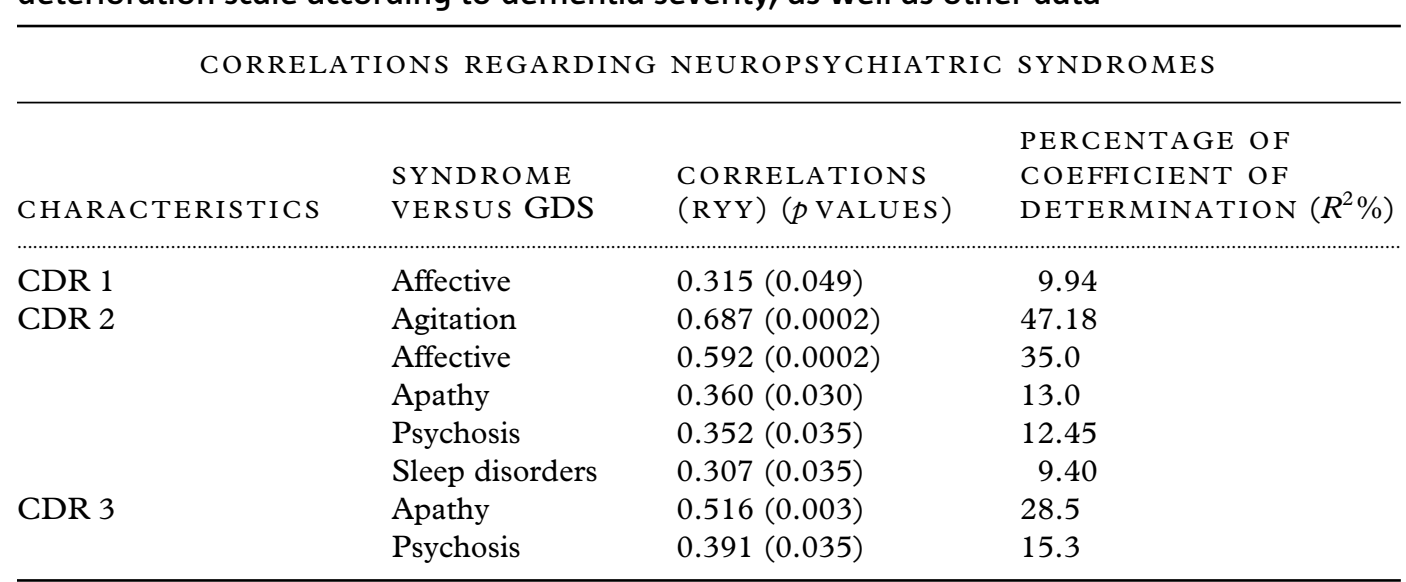

CDR: Clinical dementia rating; GDS: Global Deterioration Scale.

also showed moderate correlation. At CDR3, the apathy syndrome and agitation showed moderate correlation with GDS scores followed by the agitation syndrome. In summary, agitation, apathy, and affective were the neuropsychiatric syndromes with more substantial association with GDS scores although these correlations were moderate.

As noted earlier, the specific syndromes being tested in the current study separate sleep disturbances and apathy into two distinct syndromes. Sleep disturbances and apathy are very often disorders in $\mathrm{AD}$ patients (Robert et al., 2005; David et al., 2010), and deserve single classification as proposed by Lyketsos and Miller (2012). For example, patients with MCI presenting night-time behaviors have an increased risk for progression to dementia (Peters et al., 2013). While crucial aspects in this scenario still remain to be elucidated (e.g. overlapping psychopathological manifestations, neurobiological support for symptoms, the specific course across the disease progression, and pattern of treatment responses to specific interventions), it would not be possible to understand the potential link between $\mathrm{MCI}$ and progression into dementia without looking at sleep disturbances as a separate syndrome. Overall, the classification of psychopathological symptoms into distinct syndromes may provide methodological advantages for research designs on behavioral disturbances in AD.

Other important points to consider are the educational and cultural aspects of the population being studied. In Brazil, low educational levels require adjustments for cut-off points on the cognitive assessment of patients with dementia as reported by Brucki and Rocha (2004). In addition, cultural beliefs may interfere with the accuracy of the caregiver's report regarding specific syndromes experienced by the patient (e.g. depression or apathy). Frequently, caregivers might consider these psychopathological manifestations as part of "normal" aging (Stella et al., 2015). In the present study, we used the NPI-C in order to solve potential biases as the final scoring in this scale depends on the clinician impression (de Medeiros et al., 2010).

We note that to use both instruments the CDR and GDS - would appear to be repetitive (i.e. both measure dementia severity levels). However, we argue that it is important to analyze potential correlations between both 
scales and that such knowledge may bring a better acknowledgement of neuropsychiatric syndrome manifestations especially with regards to severity levels of dementia. More specifically, patients classified into mild dementia may exhibit some psychopathological symptoms as anxiety, apathy, or non-cooperative behavior closely related to cognitive and functional impairment (Di Iulio et al., 2010; Dillon et al., 2013; Cerejeira et al., 2012). At moderate and severe levels of dementia, patients in general have loss of spontaneous emotional reaction and diminished affective responsiveness to psychosocial situations, agitation, aggression, delusions and hallucinations, loss of communication, obsessive and repetitive behaviors, anxiety, sleep disorders, and important worsening of apathy (Di Iulio et al., 2010; Cerejeira et al., 2012), as well as aberrant vocalizations (de Medeiros et al., 2010).

In summary, the association of neuropsychiatric symptoms with cognitive decline in $\mathrm{AD}$ remains a debatable subject. Some studies have detected correlations of behavioral disturbances with cognitive impairment across the course of dementia in $\mathrm{AD}$ (Zhang et al., 2012; Dillon et al., 2013) while in others, changes in the behavioral profile of persons with $\mathrm{AD}$ were independent from decline of cognitive status suggesting partially independent mechanisms involving both conditions (Tractenberg et al., 2005). Some neuropsychiatric syndromes may worsen as the $\mathrm{AD}$ progresses, whereas others remain relatively stable even in severe stages of dementia, or exhibit fluctuating course throughout the disease deterioration. For instance, increasing severity levels of apathy have been detected over the progressive stages of $\mathrm{AD}$ (Gonfrier et al., 2012; Stella et al., 2013). Likewise, a potential non-linear trajectory impairment of cognition and behavior remains a debatable issue and may imply independent pathophysiological pathways. This would suggest that cognitive decline and behavioral disturbances could be attributed to dysfunction of distinct brain mechanisms (D'Onofrio et al., 2010; Ismail et al., 2011).

Moreover, to recognize particular phenotypic expressions and to highlight correlated brain regions underlying pathophysiological mechanisms or neural circuitry from neuropsychiatric syndromes in $\mathrm{AD}$ could provide more effective and safer pharmacological interventions for the clinical practice (Lyketsos et al., 2011; Bloniecki et al., 2015).

\section{Conclusions}

Apathy and agitation were the neuropsychiatric syndromes with the most consistent correlations with clinical global deterioration measured by the GDS. Neuropsychiatric syndromes in patients at moderate and severe levels of dementia were consistently correlated with higher GDS scores, suggesting an additional impact from these syndromes on clinical deterioration of AD.

\section{Conflict of interest}

None.

\section{Description of authors' roles}

F. Stella, J. Laks, K. de Medeiros, and O.V. Forlenza contributed to the conception and design of the study, as well as to drafting the manuscript and revising it critically for intellectual content, and assisted with writing the paper. J.S. Govone contributed to the methodological design and undertook statistical analyses.

\section{Acknowledgments}

This study was supported by the Fundação de Amparo à Pesquisa do Estado de São Paulo (FAPESP - project nos. 2009/52825-8 and 2010/18999-6). Jerson Laks is supported by Conselho Nacional de Pesquisa (CNPq) as a researcher 2, by Fundação de Apoio à Pesquisa do Estado do Rio de Janeiro (FAPERJ), and by Fundação de Empreendimentos, Pesquisa e Desenvolvimento Institucional Científico e Tecnológico do Rio de Janeiro (FEMPTEC).

\section{References}

Aalten, P. et al. (2005). The course of neuropsychiatric symptoms in dementia. Part II: relationships among behavioural sub-syndromes and the influence of clinical variables. International fournal of Geriatric Psychiatry, 20, 531-536.

Aalten, P. et al. (2008). Consistency of neuropsychiatric syndromes across dementias: results from the European Alzheimer disease consortium. Part II. Dementia and Geriatric Cognitive Disorders, 25, 1-8.

American Psychiatric Association (2000). Diagnostic and Statistical Manual of Mental Disorders, 4th edn, (DSM-IV), Text Revision. Washington, DC: American Psychiatric Association.

Bloniecki, V., Aarsland, D., Cummings, J., Blennow, K. and Freund-Levi, Y. (2015). Agitation in dementia: relation to core cerebrospinal fluid biomarker levels. Dementia and Geriatric Cognitive Disorders Extra, 4, 335-343.

Brodaty, H., Connors, M. H., Xu, J., Woodward, M. and Ames, D. For the PRIME Study Group (2015). The 
course of neuropsychiatric symptoms in dementia: a 3-year longitudinal study. Fournal of American Medical Directors Association, 16, 380-387.

Brucki, S. M. D. and Rocha, M. S. G. (2004). Category fluency test: effects of age, gender and education on total scores, clustering and switching in Brazilian Portuguese-speaking subjects. Brazilian fournal of Medical and Biological Research, 37, 1771-1777.

Cerejeira, J., Lagarto, L. and Mukaetova-Ladinska, E. B. (2012). Behavioral and psychological symptoms of dementia. Frontiers in Neurology, 3, 73. doi: 10.3389/fneur.2012.

Chi, S., Yub, J.-T., Tanc, M.-S. and Tan, L. (2014). Depression in Alzheimer's disease: epidemiology, mechanisms, and management. Fournal of Alzheimer's Disease, 42, 739-755.

Cummings, J., Jutand, M. A., Onifade, C., Richard-Harston, S. and Bourdel-Marchasson, I. (2015). Agitation in cognitive disorders: International Psychogeriatric Association provisional consensus clinical and research definition. International Psychogeriatrics, 27, $7-17$.

David, R. et al. (2010). Non-pharmacologic management of sleep disturbance in Alzheimer's disease. Fournal of Nutrition Health and Aging, 14, 203-206.

Dechamps, A., Jutand, M.A., Onifade, C., Richard-Harston, S. and Bourdel-Marchasson, I. (2008). Co-occurrence of neuropsychiatric syndromes in demented and psychotic institutionalized elderly. International fournal of Geriatric. Psychiatry, 23, 1182-1190.

de Medeiros, K. et al. For the NPI-C Research Group (2010). The neuropsychiatric inventory-clinician rating scale (NPI-C): reliability and validity of a revised assessment of neuropsychiatric symptoms in dementia. International Psychogeriatrics, 22, 984-994.

Di Iulio, F. et al. (2010). Occurrence of neuropsychiatric symptoms and psychiatric disorders in mild Alzheimer's disease and mild cognitive impairment subtypes. International Psychogeriatrics, 22, 629-640.

Dillon, C., Serrano, C. M., Castro, D., Leguizamón, P. P., Heisecke, S. L. and Taragano, F. E. (2013). Behavioral symptoms related to cognitive impairment. Neuropsychiatric Disease and Treatment, 9, 1443-1455.

D'Onofrio, G. et al. (2010). The APOE polymorphism in Alzheimer's disease patients with neuropsychiatric symptoms and syndromes. International fournal of Geriatric Psychiatry, 10, 1062-1070.

Folstein, M., Folstein, S. and McHugh, P. (1975). "Mini-Mental State": a practical method for grading the cognitive state of patients for the clinician. Fournal of Psychiatric Research, 12, 189-198.

Frisoni, G. B. et al. (1999). Behavioral syndromes in Alzheimer's disease: description and correlates. Dementia and Geriatric Cognitive Disorders, 10, 130-138.

Garre-Olmo, J., López-Pousa, S., Vilalta-Franch, J., De Gracia Blanco, M. and Bulbena Vilarrasa, A. (2010). Grouping and trajectories of the neuropsychiatric symptoms in patients with Alzheimer's disease, part I: symptom clusters. Fournal of Alzheimer's Disease, 22, 1157-1167.

Gonfrier, S., Andrieu, S., Renaud, D., Vellas, B. and Robert, P. H. (2012). Course of neuropsychiatric symptoms during a 4-year follow up in the REAL-FR cohort. Fournal of Nutrition Health and Aging, 16, 134-137.

Holthoff, V. A. et al. (2005). Regional cerebral metabolism in early Alzheimer's disease with clinically significant apathy or depression. Biological Psychiatry, 57, 512-421.

Hughes, C. P., Berg, L., Danziger, W. L., Coben, L. A. and Martin, R. L. (1982). A new clinical scale for the staging of dementia. British fournal of Psychiatry, 140, 566-572.

Ismail, Z., Minh-Quan, N., Fischer, C. E., Schweizer, T. A., Mulsant, B. H. and Mamo, D. (2011).

Neurobiology of delusions in Alzheimer's disease. Current Psychiatry Reports, 13, 211-218.

Lyketsos, C. G., Lopez, O., Jones, B., Fitzpatrick, A. L., Breitner, J. and DeKosky, S. (2002). Prevalence of neuropsychiatric symptoms in dementia and mild cognitive impairment: results from the cardiovascular health study.

Fournal of the American Medical Association, 288, 1475-1483.

Lyketsos, C. G. and Miller, D. S. For the neuropsychiatric syndromes professional interest area of the international society to advance Alzheimer's research and treatment. (2012). Addressing the Alzheimer's disease crisis through better understanding, treatment, and eventual prevention of associated neuropsychiatric syndromes. Alzheimer's $\mathcal{E}$ Dementia, 8, 60-64.

Lyketsos, C. G. et al. (2011). Neuropsychiatric symptoms in Alzheimer's disease. Alzheimer's \& Dementia, 7, 532-539.

McKhann, G., Drachman, D., Folstein, M., Katzman, R., Price, D. and Stadlan, E. M. (1984). Clinical diagnosis of Alzheimer's disease: report of the NINCDS-ADRDA work group under the auspices of department of health and human services task force on Alzheimer's disease. Neurology, 34, 939-944.

Mirakhur, A., Craig, D., Hart, D. J., McLlroy, S. P. and Passmore, A. P. (2004). Behavioural and psychological syndromes in Alzheimer's disease. International fournal of Geriatric Psychiatry, 19, 1035-1039.

Peters, M. E. et al. (2013). Neuropsychiatric symptoms as risk factors for progression from CIND to dementia: the cache county study. American fournal of Geriatric Psychiatry, 21, 1116-1124.

Pfeffer, R. I., Kurosaki, T. T., Harrah, C. H. Jr., Chance, J. M. and Filos, S. (1982). Measurement of functional activities in older adults in the community. fournal of Gerontology, 37, 323-329.

Reisberg, B., Ferris, S. H. and De Leon, M. J. (1982). The global deterioration scale for assessment of primary degenerate dementia. American foumal of Psychiatry, 139, 1136-1139.

Robert, P. H. et al. (2005). Grouping for behavioral and psychological symptoms in dementia: clinical and biological aspects. Consensus paper of the European Alzheimer disease consortium. European Psychiatry, 20, 490-496.

Robert, P. H. et al. (2009). Proposed diagnostic criteria for apathy in Alzheimer's disease and other neuropsychiatric disorders. European Psychiatry, 24, 98-104.

Spalletta, G. et al. (2004). Cognition and behaviour are independent and heterogeneous dimensions in Alzheimer's disease. Fournal of Neurology, 251, 688-695.

Spalletta, G. et al. (2010). Neuropsychiatric symptoms and syndromes in a large cohort of newly diagnosed, untreated 
patients with Alzheimer disease. American fournal of Geriatric Psychiatry, 18, 1026-1035.

Stella, F. et al. (2013). The Brazilian version of the Neuropsychiatric Inventory-Clinician Rating Scale (NPI-C): reliability and validity in dementia. International Psychogeriatrics, 25, 1503-1511.

Stella, F. et al. (2015). Caregiver report versus clinician impression: disagreements in rating neuropsychiatric symptoms in Alzheimer's disease patients. International Fournal of Geriatric Psychiatry, early view, doi: 10.1002/gps.4278.

Tractenberg, E. R., Weiner, M. F., Cummigns, J. L., Patterson, M. B. and Thal, L. J. (2005). Independence of changes in behavior from cognition and function in community-dwelling persons with Alzheimer's disease: a factor analytic approach, fournal of Neuropsychiatry $\mathbb{E}$ Clinic Neurosciences, 17, 51-60.

Van der Mussele, S. et al. (2015). Agitation-associated behavioral symptoms in mild cognitive impairment and Alzheimer's dementia. Aging Eo Mental Health, 19, 247-257.

Zhang, M., Wang, H. and Yu, T. L. (2012). Prevalence of neuropsychiatric symptoms across the declining memory continuum: an observational study in a memory clinic setting. Dementia and Geriatric Cognitive Disorders Extra, 2, 200-208. 